\title{
Blood to cerebrospinal fluid human chorionic gonadotropin-beta ratios in intracranial germ cell tumors
}

\author{
Paul B. Rogers, M.R.C.P., F.R.C.R., Eliot C. Sims, M.R.C.P., and Nicholas Plowman, M.D., \\ F.R.C.P., F.R.C.R.
}

Department of Radiotherapy, St. Bartholomew's Hospital, London, United Kingdom; and Department of Haematology and Oncology, The Hospital for Sick Children, London, United Kingdom

Levels of human chorionic gonadotropin-beta (HCG-beta) are elevated in up to $43 \%$ of patients with intracranial germ cell tumors (GCTs) and are useful in the diagnosis of these tumors and the follow up of such patients. The ratio of blood HCG-beta to lumbar cerebrospinal fluid (CSF) HCG-beta in these patients at presentation has not been defined. Twenty-two patients with intracranial GCTs have been treated at St. Bartholomew's Hospital over the past 15 years. Two (17\%) of 12 germinomas and seven (70\%) of 10 nongerminomatous GCTs had elevated blood HCG-beta at presentation. Four cases of pineal region GCTs (one of 12 germinomas and three of 10 nongerminomatous GCTs) had paired, elevated, blood and lumbar CSF HCG-beta levels. The mean blood to CSF ratio was 1:10 (range 1.7-18.4), which is substantially lower than the ratio of 286:1 reported in systemic GCTs. The authors confirm the finding of a previous single report showing that ventricular CSF HCG-beta sampling via an accessible ventriculoperitoneal shunt reservoir may give a spuriously negative result, and they discuss the pathophysiology of the blood-brain barrier in the pineal region and the implications of the intrathecal administration of chemotherapy.

Key Words * pineal gland * intracranial germ cell tumor * human chorionic gonadotropin-beta concentration * blood-brain barrier

Primary intracranial germ cell tumors (GCTs) are not only rare, accounting for 1 to $2 \%$ of primary brain tumors, but those that secrete the tumor markers human chorionic gonadotropin-beta (HCG-beta) and alpha-fetoprotein (AFP) are even more scarce. Human chorionic gonadotropin-beta is produced by germinomas or choriocarcinomatous elements of nongerminomatous GCTs and has a half-life of up to 48 hours; AFP is produced by yolk sac elements of nongerminomatous GCTs and has a half-life of 5 to 6 days. We reviewed 19 studies that showed that between $0 \%$ and $43 \%$ of patients with intracranial GCTs had elevated HCG-beta, but only nine patients in total[2,4,5,11] had paired blood and cerebrospinal fluid (CSF) HCG-beta data with ratios ranging from 6:1 to 17:7. Only two other teams of authors have commented on the ratio; Allen, et al.,[2] described four patients with intracranial nongerminomatous GCTs whose blood-to-lumbar CSF ratios at presentation were 1:18, 6:1, 1:14, and 1:6, respectively. One 
of these patients, with a high level of lumbar CSF HCG-beta had a very low ventricular fluid level. Edwards, et al.,[7] also looked at blood and CSF markers in pineal nongerminomatous GCTs, concluding nonspecifically that blood levels are usually lower than spinal fluid levels and that a reversed ratio suggests systemic disease.

These tumors markers can be extremely useful in diagnosis, serial evaluation of response to therapy, and diagnosis of persisting active disease in nongerminomatous GCTs[9] (germinomatous GCT may also secrete low levels of HCG-beta). It is now well established that nongerminomatous GCTs are much more difficult to cure than intracranial germinomas.[6] Sano[12] has clearly demonstrated how poor the prognosis was for patients with intracranial choriocarcinoma undergoing standard treatment protocols. An understanding of the ratio of HCG-beta in blood and CSF and the pathophysiological mechanisms of the blood-brain barrier (BBB) at this site may help further improve patient outcome by earlier detection and different treatment strategies.

\section{CLINICAL MATERIAL AND METHODS}

Over the past 15 years, 22 patients with intracranial GCTs have been treated at St. Bartholomew's Hospital. Twelve patients (nine male and three female) had germinomas, and their median age was 18 years. Six patients had tumors located in the pineal region, five in the suprasellar, and one in both sites. Ten patients (eight male, two female) had nongerminomatous GCTs (a term incorporating malignant teratoma, embryonal carcinoma, yolk sac tumor [also known as endodermal sinus tumor], choriocarcinoma, and mixed GCTs), and their median age was also 18 years. Seven tumors were of pineal origin, but one also had suprasellar disease and another had left frontal lobe involvement. Two tumors originated in the suprasellar region and one in the fourth ventricle. The treatment technique and outcomes have previously been reported.[10,13,14] We reviewed the concentrations of HCG-beta in blood and CSF in these patients. Lumbar puncture for CSF tumor marker levels had been carried out only after ventriculoperitoneal (VP) shunt insertion where raised intracranial pressure had been present. The ratios of blood HCG-beta to lumbar CSF HCG-beta were calculated. The gradient across the BBB was compared with data from systemic nongerminomatous GCT. We discuss the function of the BBB in relation to the pineal gland and possible implications for intrathecal delivery of chemotherapeutic agents.

\section{RESULTS}

\section{Germinomas}

At presentation, two (17\%) of the 12 patients who both had tumors located in the pineal region had elevated blood HCG-beta concentrations of 32 and $21 \mathrm{IU} / \mathrm{L}$, respectively (normal lt $2 \mathrm{IU} / \mathrm{L}$ ). Only the latter case had a paired CSF level measured, and this was $140 \mathrm{IU} / \mathrm{L}$, giving a blood-to-lumbar CSF ratio of 1:6.7. The other 10 patients had negative blood HCG-beta levels. One boy, who had a negative blood HCG-beta level, underwent lumbar CSF measurement after VP shunt insertion, and this was negative for HCG-beta and AFP. No patients with germinoma, by definition, had elevated AFP.

\section{Nongerminomatous GCTs}

At presentation, seven (70\%) of the 10 patients with nongerminomatous GCTs had elevated blood levels of HCG-beta. Four of these patients had pineal disease, one had both pineal and suprasellar tumors, and two had suprasellar disease. The median level was 723 IU/L (range 19-1431). Three of the 10 patients with nongerminomatous GCT (all with pineal disease) had paired elevated HCG-beta levels. One patient with negative blood HCG-beta also had a negative CSF HCG-beta. One patient with a pineal 
nongerminomatous GCT, who underwent VP shunt insertion, had a CSF level of 17 IU/L, but no blood level was documented. One patient had an intratumoral cyst-fluid HCG-beta concentration of 1457 IU/L but did not have the CSF levels documented and only had a blood level of $3 \mathrm{IU} / \mathrm{L}$. There were four patients (40\%) with elevated blood AFP (ranging from 126-112,500 IU/L) but no CSF levels for comparison.

\section{Ratios of Blood to Lumbar CSF HCG-beta}

The results of the paired blood and CSF HCG-beta data are as follows. Case 1, a 14-year-old boy with germinoma located in the pineal region, had a blood HCG-beta level of $21 \mathrm{IU} / \mathrm{L}$ and a lumbar CSF level of $140 \mathrm{IU} / \mathrm{L}$, giving a ratio of 1:6.7. Case 2, a 14-year-old boy with a pineal region nongerminomatous GCT, had a blood HCG-beta level of $19 \mathrm{IU} / \mathrm{L}$ and a lumbar CSF level of $350 \mathrm{IU} / \mathrm{L}$, giving a ratio of 1:18.4. In this patient the lateral ventricular CSF was sampled prior to therapy (accessed via the VP shunt), and this CSF HCG-beta result was negative despite the elevated lumbar CSF level. Case 3, a 17-year-old boy with a pineal region nongerminomatous GCT, had a blood HCG-beta level of 1431 IU/L and a lumbar CSF level of 17,500 IU/L, giving a ratio of 1:12.2. Case 4, a 19-year-old man with a pineal region nongerminomatous GCT, had two blood HCG-beta levels during diagnostic work up of $240 \mathrm{IU} / \mathrm{L}$ and $723 \mathrm{IU} / \mathrm{L}$ with paired lumbar CSF levels of $409 \mathrm{IU} / \mathrm{L}$ and $1390 \mathrm{IU} / \mathrm{L}$ at these times. The ratios were $1: 1.7$ and $1: 1.9$, respectively.

The lumbar CSF HCG-beta concentrations ranged from 140 to 17,500 IU/L, and the blood levels ranged from 19 to $1431 \mathrm{IU} / \mathrm{L}$. The mean ratio of lumbar CSF to blood HCG-beta was 1:10 (range 1:1.7-1:18.4).

\section{DISCUSSION}

\section{Blood-Brain Barrier in the Pineal Region}

The observation of a 10-fold ratio (range 1.7-18.4) blood to lumbar CSF for HCG-beta levels in this disease at diagnosis is of interest. First, it implies that evaluation of lumbar CSF HCG-beta is 10 times more sensitive than blood HCG-beta in detecting intracranial GCT. In our series $70 \%$ of nongerminomatous GCTs and $17 \%$ of germinomas had elevated HCG-beta in blood. Although this is a higher proportion of nongerminomatous GCT than in other series, it is likely, based on our data, that some of the patients with negative blood levels had mildly elevated CSF levels. Therefore it underscores the importance of lumbar CSF sampling, when safe, in diagnosing tumors in the pineal or suprasellar regions. Second, this 10-fold ratio implies that the BBB is deficient in the pineal region when compared with the ratio of systemic germ cell blood to lumbar CSF[3] of 286:1. Some investigators have postulated the pineal site to be disengaged from the BBB,[8] along with the neurohypophysis, the median eminence (pituitary stalk and adjacent floor of the third ventricle), the organum vasculosum of the lateral terminalis, and the area postrema--points of interest in modern neuroradiology, as these structures may show contrast enhancement, unexpected if they were beyond the BBB. If this were so, there should be no difference between the blood and CSF levels. Tight junctions between the capillary endothelial cells account for the BBB and prevent the passive transfer of even many small molecules into the extracellular space and CSF. Fat-soluble molecules are the only compounds that easily enter the brain across this endothelial barrier. Within the choroid plexus, a blood-CSF barrier occurs at the level of the epithelial cells, rather than at the endothelium of the choroidal vessels, although in this paper we refer to the whole system as the "blood brain barrier."

Some authors assert that the response of pineal GCTs to chemotherapy is attributable to the deficient or 
absent BBB in this region. This is indeed a possibility, but, if so, one might expect HCG-beta secretion by the tumor to have equal access to the blood. Analysis of our data has shown definitively that this is not so. At presentation in this disease, there is a gradient (mean 10-fold) of blood to lumbar CSF of HCG-beta concentrations. In systemic GCTs, Bagshawe and Harland[3] showed clearly that there was a 286-fold (range 64-1546-fold for choriocarcinoma and 104-560-fold for teratoma) gradient for blood to CSF. Furthermore, they showed that a ratio of less than 60:1 predicted development of cerebral metastases. It is of interest that the ratio of 1:10 is substantially lower than the reversed ratio of 286:1 found in systemic choriocarcinoma. This suggests to us that the pineal glandular-HCG-beta secretion does have more privileged access to the blood than would be predicted from passage across the BBB.

\section{Lateral Ventricular HCG-beta Route of Chemotherapy Administration}

In a patient with a VP shunt, the documentation of lumbar CSF to lateral ventricular CSF HCG-beta values of 353:0 IU/L is extremely interesting. There is one other case demonstrating this phenomenon in the literature.[2] This indicates that countercurrent diffusion is extremely small (despite a functioning shunt, which might be thought to decrease the CSF current flow out of the lateral ventricles through the third to the fourth ventricle, that is, caudally past the pineal gland). This also explains why blood levels of HCG-beta are not higher than expected from the gradient data presented in VP shunt patients; that is, the lateral ventricular CSF HCG-beta concentrations are sufficiently low as to not "boost" the blood HCG-beta values via the shunt to the peritoneum and hence blood. Indeed, in Case 2, the blood HCG-beta level was $19 \mathrm{IU} / \mathrm{L}$ when the lateral ventricular CSF HCG-beta level was unrecordable (and lumbar HCG-beta was $353 \mathrm{IU} / \mathrm{L}$ ). This case of a spuriously negative ventricular HCG-beta concentration suggests that lumbar CSF sampling should be considered after VP shunt insertion in patients with pineal region tumors.

These observations also have relevance to the intrathecal administration of chemotherapy via the lumbar puncture route. Although intrathecal administration of methotrexate by the lumbar route seems to have better access to the lateral ventricles in pharmacokinetic studies in primates[1] than HCG-beta from the data presented here, the poor access to all intracranial sites has been demonstrated in childhood acute lymphoblastic leukemia by clinical central nervous system relapse data.[15] Furthermore, this has been the reason for cranial irradiation prophylaxis or very high-dose systemic methotrexate to boost intracranial drug levels in current childhood acute lymphoblastic leukemia protocols. The spuriously low, or negative, ventricular HCG-beta is consistent with the rarity of abdominal spread via the VP shunt in intracranial GCT. We have previously reported one of the four patients with an AFP-secreting nongerminomatous GCT who was found to have peritoneal relapse via the shunt after a routine blood AFP level became elevated.[13] Our limited data would support the idea that there might be a pharmacokinetic advantage in administering adjuvant or therapeutic intrathecal chemotherapy for nongerminomatous GCT via a lateral ventricular reservoir, in addition to standard intravenous chemotherapy, as this would ensure adequate ventricular concentrations and may help prevent peritoneal spread, as well as improve local control. Further data are required and from a large study such as the International Society for Pediatric Oncology intracranial GCT study.

Finally, we wonder if peritoneal seeding is more likely in primary suprasellar GCTs as they are closer to the shunt with a shorter "countercurrent diffusion"-path length to travel. Similarly, the aforementioned may provide the explanation as to why the 5 to $7 \%$ risk of systemic dissemination of cerebellar medulloblastoma seems, in the St. Bartholomew's/Great Ormond Street and other clinicians' series, to be unrelated to the presence of a VP shunt. The cells in this situation would have to undergo countercurrent 
diffusion back up the sylvian aqueduct in addition to the course needed for pineal GCT cells to reach the lateral ventricular fluid.

\section{CONCLUSIONS}

The lumbar CSF is the most sensitive accessible measurement of tumor marker levels in HCG-beta secreting pineal GCT available to clinicians. That a tumor cyst contains the highest HCG-beta concentrations should be no surprise, but this has no use as a serial marker. Lateral ventricular CSF levels are spuriously low and may be misleading in detecting and assessing tumor response unless followed by lumbar puncture (after VP shunt insertion if there is a risk of coning). The observed ratio of blood to lumbar CSF is only of the order of 1:10, out of keeping with the $286: 1$ ratio observed for systemic choriocarcinoma and demonstrating neatly that the pineal region is, to some extent, disengaged from the BBB.

\section{References}

1. Abelson HT, Ensminger W, Rosowsky A, et al: A primate model for the study of CNS pharmacokinetics anti-neoplastic agents, in Whitehouse JMA, Kay HEM (eds): CNS Complications of Malignant Disease. Baltimore: University Park Press, 1979, pp 397-406

2. Allen JC, Nisselbaum J, Epstein F, et al: Alphafetoprotein and human chorionic gonadotropin determination in cerebrospinal fluid. J Neurosurg 51:368-374, 1979

3. Bagshawe KD, Harland S: Immunodiagnosis and monitoring of gonadotrophin-producing metastases in the central nervous system. Cancer 38:112-118, 1976

4. Baranzelli MC, Patte C, Bouffet E, et al: Nonmetastatic intracranial germinoma: the experience of the French Society of Pediatric Oncology. Cancer 80:1792-1797, 1997

5. Bridgewater JA, Souhami RL, Allgrove J, et al: Intracranial germ cell tumours presenting with hypopituitarism. Successful treatment with chemotherpay alone. Eur J Cancer 30A:1401-1403, 1994

6. Dearnaley DP, A'Hearn RP, Whittaker S, et al: Pineal and CNS germ cell tumors: Royal Marsden experience 1962-1989. Int J Radiat Oncol Biol Phys 18:773-788, 1990

7. Edwards MSB, Hudgins RJ, Wilson CB, et al: Pineal region tumors in children. J Neurosurg 68:689-697, 1988

8. Ganong WF: Review of Medical Physiology, ed 11. Lang, Los Altos, CA: 1983, pp 496-513

9. Kaye SB, Bagshawe KD: Chemical markers in spinal fluid for tumours of the central nervous system (CNS), in Whitehouse JMA, Kay HEM (eds): CNS Complications of Malignant Disease. Baltimore: University Park Press, 1979, pp 306-323

10. Plowman PN, Kingston JE, Sebag-Montefiore D, et al: Clinical efficacy of perceived 'CNS-friendly' chemoradiotherapy for primary intracranial germ cell tumours. Clin Oncol 9:48-53, 1997

11. Rustin GJS, Newlands ES, Bagshawe KD, et al: Successful management of metastatic and primary germ cell tumours in the brain. Cancer 57:2108-2113, 1986

12. Sano K: So-called intracranial germ cell tumors: are they really of germ cell origin? Br $\mathbf{J}$ Neurosurg 
9:391-401, 1995

13. Sebag-Montefiore DJ, Douek E, Kingston JE, et al: Intracranial germ cell tumours. I. Experience with platinum based chemotherapy and implications for curative chemoradiotherapy. Clin Oncol 4:345-350, 1992

14. Sebag-Montefiore DJ, Doughty D, Plowman PN: Intracranial germ cell tumours. II. The application of a partial transmission block technique to reduce late morbidity. Clin Oncol 4:351-354, 1992

15. Simone JV, Hustu HO, Aur RJA: Prevention and treatment of central nervous system leukaemia in childhood, in Whitehouse JMA, Kay HEM (eds): CNS Complications of Malignant Disease. Baltimore: University Park Press, 1979, pp 19-35

Manuscript received May 4, 1998.

Accepted in final form June 24, 1998.

Address reprint requests to: P. Nicholas Plowman, M.D., Consultant Clinical Oncologist, St. Bartholomew's Hospital, West Smithfield, EC1A 7BE London, United Kingdom. 